\title{
Multitask Aspect_Based Sentiment Analysis with Integrated Bidirectional LSTM \& CNN Model.
}

TRANG UYEN TRAN*

The Vietnam-Korea University of Information and Communication Technology, Danang University, Vietnam

HA THANH THI HOANG

The University of Economic, Danang University, Vietnam

PHUONG HOAI DANG

The University of Science and Technology, Danang University, Vietnam

MICHEL RIVEILL

Equipe Maasai, Inria Sophia-Antipolis, Laboratoire I3S, UMR CNRS 7271, Universite' Côte d'Azur, Nice, France

Sentiment analysis or opinion mining used to understand the community's opinions on a particular product. Sentiment analysis involves building the opinion collection and classification system. Aspect-based sentiment analysis focuses on the ability to extract and summarize opinions on specific aspects of entities within sentiment document. In this paper, we propose a novel supervised learning approach using deep learning techniques for multitask aspect-based opinion mining system that support four main subtasks: extract opinion target, classify aspect-entity (category), and estimate opinion polarity (positive, neutral, negative) on each extracted aspect of entity. Using extra POS layer to identify morphological features of words combines with stacking architecture of BiLSTM and CNN with word embeddings achieved by training GloVe on Restaurant domain reviews of the SemEval 2016 benchmark dataset in our proposed method is aimed at increasing the accuracy of the model. Experimental results showed that our multitask aspect-based sentiment analysis model has extracted and classified main above subtasks concurrently and achieved significantly better accuracy than the state-of-the-art methods.

CCS CONCEPTS • Machine Learning • Machine Learning approachs • Neural Networks

Additional Keywords and Phrases: Aspect-Based Sentiment Analysis, Multitask, BiLSTM, CNN, Word embedding, POS tag

\section{INTRODUCTION}

The sentiment analysis [2] is a field of research on the ability to extract and categorize opinions of the community about entities and attributes related to these entities such as products and services...that support for tracking the mood of the community. Therefore, the need to exploit the ability to detect and understand the views automatically is increasingly interested in research. The standard opinion mining focus on sentiment of the overall document or sentence. However, in case of customer reviews, they always discuss about many aspects of each entity in the sentence or document and present the sentiment on every single aspect of entity rather than expressing the sentiment on whole document. Because of this reason, we focus on Aspect-Based Sentiment Analysis (ABSA) with the purpose to identify aspect of entity in sentiment text and the opinion presented on each aspect. The innovations of proposed ABSA models, the existing aspect extraction and opinion specification techniques are absolutely the core research directions of sentiment analysis.

* Place the footnote text for the author (if applicable) here. 
In this paper, we propose to apply the deep learning approach for multitask ABSA using an integrated CNNBiLSTM architecture with word embeddings [30] obtained by training GloVe [16] on reviews of the SemEval 2016 dataset. The reasons for our choosing model are that: (i) CNN with various-size convolution kernel mechanism can support effectively to extract local features (ii) LSTM can well process long sequences based on the ability to keep long-term memory and control the gradient backpropagation through time to solve the gradient vanishing and exploding issue. The experimental results show that our proposed model used for ABSA proved to be more effective than previous state-of-the-art approaches.

In the rest of this paper, we organize as follows. Section 2 presents the related works for ABSA. Our approach for proposed model with BiLSTM and CNN integrated architecture is described in Section 3. Section 4 presents our experiments using a benchmark dataset and discussion about received results. Finally, Section 5 focuses on our conclusion and future research direction.

\section{RELATED WORKS}

Most of recent sentiment analysis researches have focused on two main approaches [2]: (i) lexicon-based approach with two sub classifications dictionary-based and corpus-based using class of measures on various models to calculate the opinion orientation for the document from the opinion orientation of words in the opinion text. This approach identifies the sentiment polarity of words based on the correspondence between sentiment words in opinion lexicon and the data (ii) machine learning-based approach using classification techniques to classify text, identify opinion on training and testing data with supervised learning or on unlabeled data with unsupervised learning.

In lexicon-based approach, [19][20] used a list of seed words in the dictionary to identify semantic orientation for adjectives in the document. [23] considered the relationship between phrases and a set of positive or negative seed word to correct the semantic of these words with the two statistical measurements PMI and LSA for calculating the average semantic orientation of all phrases to receive the sentiment. WordNet was used in [15] to determine the shortest route between words in the document and the two seed words "good" and "bad" to predict the sentiment orientation of the words. [9] applied the k-means clustering algorithm and TF-IDF to cluster opinion texts into one of two groups: positive or negative group. [36] identified the orientation of opinion document based on the context dependent technique. [10] used the syntactic relationship between opinion and aspect with a small set of opinion seed words to extract concurrent opinions and aspects. [17] developed thesauruses dictionary of words to build the Semantic Orientation Calculator for labeling the positive or negative orientation to the sentiment document. [24] applied the Mutual Information and Chi square for feature extraction and used Unigram Naive Bayes model to identify the polarity of the tweet.

Sentiment analysis based on machine learning-based approach is also recently interested. [34] extracted aspects and opinion phrases by learning patterns with lexicalized HMM model. [8] extract both features and opinions by combining two CRF variations: Skip-CRF and Tree-CRF. [28] trained SVM classifier to correct aspect terms and also identify the sentiment expressed on these aspects of reviews. [11] applied a hierarchical deep learning framework with Recursive Neural Network, Matrix-Vector Recursive Neural Network and Recursive Neural Tensor Network to extract aspects and detect opinion polarities that expressed on them. [29] presented PhraseRecursive Neural Network to predict the sentiment of the aspect in document. This model is the extention of RNN that use dependence and constituent trees of each sentence in the text to get the syntactic information. [6] proposed Target-Dependent LSTM model to detect the sentiment polarity towards the target based on the target word and context word. [18] expanded CRF model using an adding neural layer between input and output nodes to apply a continuous word embedding mechanism for extracting both relevant aspects and opinions. [35] used an integrated model of recursive neural network and CRF to extract aspects and opinions. [39] proposed an attention-based LSTM model for ABSA having ability to capture the key part of sentence concerning to each given aspect. A deep memory network model was applied by [7] to pick up the worth of context word with a few computational layers to calculate the important degree of context word with each of these layers is a neural attention network. [4] used an Interactive Attention Networks including two attention networks that focus on the important parts in the aspect and context to model the aspect and context interactively. [22] used a Recurrent Attention Network on memory to apply multiple attention mechanism for 
synthesizing dispersed opinion features in the sentence for finding the sentiment of aspects in customer reviews. Liu et al. [25] proposed an attention based model by combining content attention mechanism and context attention mechanism to gain the key information of aspects and take both the order of words and their relations for ABSA. [26] proposed two solutions for enhancing the productivity of attention mechanism on attention-based LSTM model: one for better capturing the aspect semantics of target word by a new encoding technique for target and one for applying the syntactic information to built a syntax-based attention model. Gated Convolutional Networks with the integration of gating mechanisms and CNN was proposed by Xue and Li [33]. The authors presented parallel computations on training model based on time independency of convolutional layers and gating units independent action mechanism. Trang et al. [31][32] proposed two integrated BiGRUCRF and Bi-IndyLSTM-CRF approachs for extracting aspects in opinion document. They took advantage of the forward and backward mechanism of Bi-IndyLSTM and BiGRU for their models using the previous and the next token to extract features with higher accuracy. Based on deep learning approach, we propose a multitask ABSA and have achieved better accuracy on both the F1-score and Accuracy-score than the previous approaches.

\section{METHODOLOGY}

In this paper, we apply two stacked models with CNN and BiLSTM for multitask ABSA. The integrated approach with the extra POS tag layer allow optimal use of the capabilities of CNN and BiLSTM. CNN has ability to get the local feature around a mentioned word in input sentence but it is not possible to combine distant information of the current word. Nevertheless, BiLSTM with its capacity can combined locally extracted features by the CNN layer around each word at current position with extracted features of words at distant previous and next position. In addition, the BiLSTM on top of CNN also serves as a classifier to infer other components that need to be extracted simultaneously. This integrated approach is aimed at increasing model accuracy in multitask execution : opinion targets, categories (entities-aspects) and sentiments extraction.

\subsection{Convolutional Neural Network (CNN)}

Although CNN were originally useful for image processing, they have been recently applied to several natural language processing tasks and got achievement [3][27][40]. When working with sequences of words, convolutions apply the ability to extract local features around each word. The input sentence with length $\mathrm{N}$ $\left(w_{1}, w_{2}, \ldots, w_{N}\right)$ after going through the embedding layer composed of POS tag layer to define the morphology of words $\left(e_{1}, e_{2}, \ldots, e_{N}\right)$ will be feed to $\mathrm{CNN}$ for extracting high level features. The feature output is as input of the next layer_BiLSTM.

\subsection{Bidirectional Long Short-Term Memory (BiLSTM)}

LSTM is one of the variants of Recurrent neural network (RNN)_a deep learning technique that has been recently used for sequential learning tasks. RNN has ability to model sequences of arbitrary length. This capacity is due to repetition of a recurrent unit along tokens in the sequence. However, RNN is limited because of the issues involved in vanishing and exploding gradients [41] that made it is not sufficient for learning longterm dependencies. Because of that, LSTM was applied to solve these weak points and it can be depicted with three gates mechanism as follows: forget gate layer $f_{t}$ for filtering and deciding which information to forget from the cell state $C_{t}$, input gate layer $i_{t}$ for deciding which values to update and output gate layer $o_{t}$.

A main issue of unidirectional LSTM is that it allows learning representations from previous time steps. Thus, it only preserves information of the past because the only inputs it has seen are from previous time steps. In some cases, we should use Bidirectional LSTM (BiLSTM) to learn representations from future time steps as well to better understand the context and eliminate the ambiguity incurred by learning one way.

\subsection{Data Preprocessing}

Data cleaning: In this task, we remove unusual characters and emoticons; convert all words to lowercase, remove numbers (i.e. '8') and empty sentences; find and replace slang or abbreviated with word substitutions (i.e. 'u' -> 'you'); lemmatize all words into their base form (e.g. 'computing', 'computed' -> compute). 
Word embedding: We use pre-trained word vectors from GloVe [16], an unsupervised learning technology, for learning word representation. The purpose of training is to use statistical information to find similarities among words.

Part-of-Speech (POS) tags: We generate POS tags for words by Stanford POS Tagger to assign parts of speech to each word in input review.

IOB2 tags: Generating IOB2 tags on the top of our model to label I/O/B to words in review is intended to extract opinion targets. In particular, we consider the opinion target extraction as a word labeling task. According to IOB2, each word in review can get one of three tags $\mathrm{B} / \mathrm{I} / \mathrm{O}$ and can be confirmed as following rules:

the first word label in review should start with "B-" or "O", not "I-", the valid patterns should be "O B-label", "B Ilabel" but "O I-label" is invalid. The first label of one opinion target, maybe word or phrase, should start with "B", not "I-".

\subsection{Proposed Multitask Aspect-based Sentiment Analysis model}

\subsubsection{Deep CNN-IOB2 model for extracting opinion target}

The data after being cleaned according to the sequence of steps in 3.3 was applied for the GloVe embedding layer with 100-dimension feature vector to encode all properties related to the semantics and syntax of each word in input review. Each input sentence with length / will be fixed to the maximum sentence length of the dataset. We use 0 s padding scheme for shorter sentence. This helps ensure all sentence matrices have the same dimensions. At the same time, generating POS tagger to identify the parts of speech of word will make the 34-dimention feature vector. Next, we concatenate the two types of vectors above resulted from GloVe layer and POS tagger and feed for the deep three-layer CNN architecture to extract features.

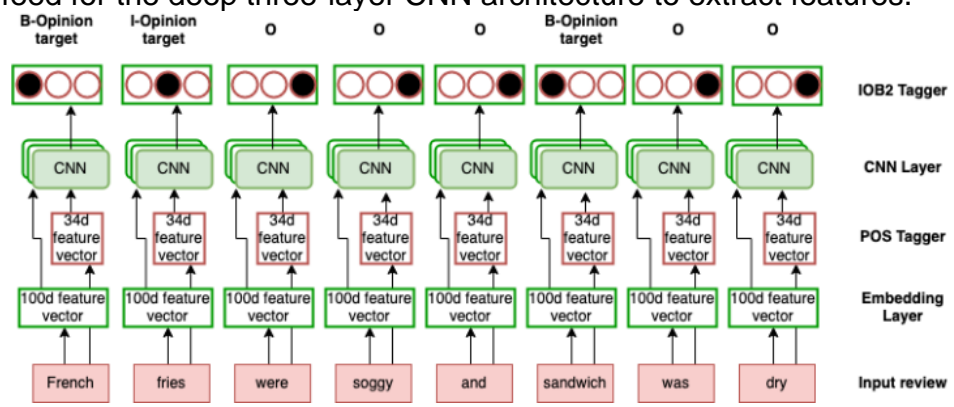

Figure 1: Deep CNN-IOB2 model for extracting opinion target

Using the IOB2 scheme on top of the CNN layers aims to label each token with one of three tags B///O with $B$ and I represent the opinion target at the beginning and the next/inside position of the target word phrase respectively and $O$ represents the outside/irrelevant word. By applying this above labeling mechanism we can achieve the opinion target extraction task.

\subsubsection{Multitask Aspect-based Sentiment Analysis (MABSA) model}

We apply data preprocessing, GloVe word embedding, POS tag and feature extraction steps like the above deep CNN-IOB2 model and add some more elements to extract the remaining three components in the mentioned multitask extraction process.

Using the BiLSTM layer on top of the CNN we can take advantage (i) learning ability from long-term memory in previous time step of LSTM and (ii) information preserve ability from both sides: past and future of the current time step based on bidirectional mechanism. Next, we apply the output of the BiLSTM to the average pooling layer to reduce the number of dimensions of word vectors in sentence review. We use a fully-connected layer (dense) in the final stage of the proposed model and feed the output to the softmax layer to compute propability distribution over the possible categories for completing classifying and extracting what we are looking for: the category (entity-aspect) and sentiment simultaneously. Our proposed model has achieved remarkable accuracy compared to previous state-of-art models. 


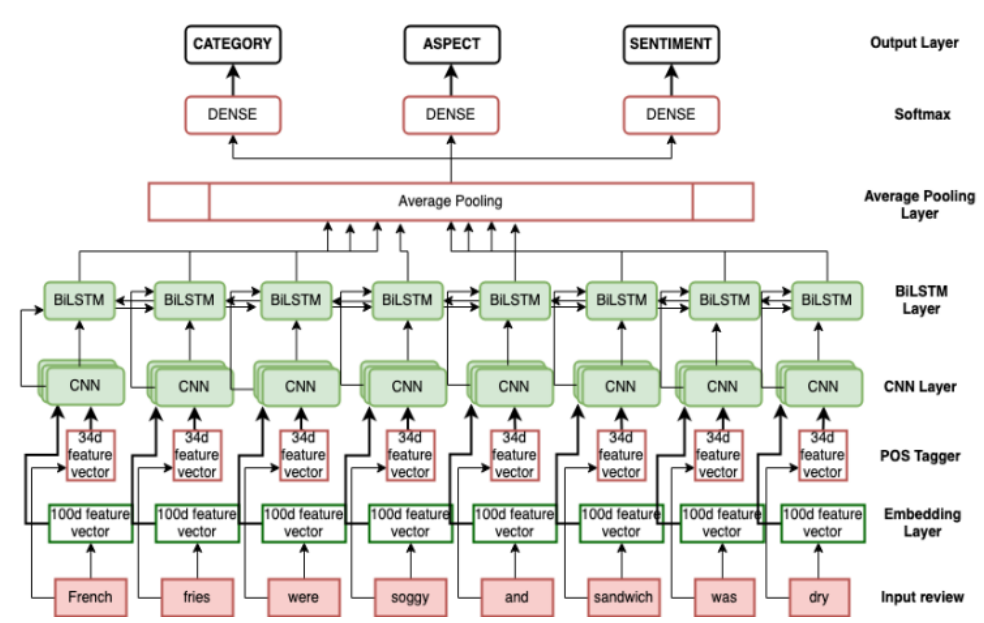

Figure 2: Deep Multitask Aspect-based Sentiment Analysis model for classifying three remaining subtasks

\section{EXPERIMENTS AND RESULTS}

\subsection{Dataset}

We use the SemEval2016 Task 5 benchmark datasets ${ }^{1}$ as a corpora for training and evaluation. Our Deep CNNIOB2 model and Multitask Aspect-based Sentiment Analysis model have been tested on reviews from the Restaurant domain and has initially given feasible results.

Table 1: Using Restaurant domain in the SemEval 2016 dataset for experiment

\begin{tabular}{llll}
\hline Dataset & Domain & $\begin{array}{l}\text { Train/Test } \\
\text { Sentences }\end{array}$ & $\begin{array}{l}\text { Train/Test } \\
\text { opinion_targets }\end{array}$ \\
\hline SemEval 2016 & Restaurant & $2000 / 676$ & $1743 / 622$ \\
\hline
\end{tabular}

\subsection{Experimental results}

We perform experiments for our proposed models in the Restaurant domain of the SemEval 2016 dataset and evaluate our models' performance using accuracy value and F1-score, the same as the previous baseline methods. We use pretrained GloVe word embeddings with 100-dimensional feature vector, concatenate with 34-dimensional POS tag vector for the feature extracting CNN layer, the maximum input sequence length is 62 , the rmsprop optimizer with learning rate 0.001 , dropout 0.25 and a batch size of 128 in the two models. The number of epochs is set to 125 and 300 for the opinion target extracting CNN-IOB2 model and the Multitask Aspect-based Sentiment Analysis model on Restaurant domain respectively. Experiment results of our models are presented in Table 2 and Table 3 below.

Our proposed Deep CNN-IOB2 model for extracting opinion target words or phrases of input reviews is evaluated through comparison with the following baseline models also experimented on the same benchmark dataset_the SemEval 2016, Restaurant domain. Besides, our Multitask Aspect-based Sentiment Analysis model of classifying the remaining subtasks such as category, aspect and sentiment polarity also achieved remarkable results.

Table 2: Comparison results in F1_score and Accuracy_score on Restaurant domain of the SemEval 2016 dataset

\begin{tabular}{lll}
\hline Model & Opinion target extraction & \\
\hline & F1_score & Accuracy_score \\
NLANGP(U) [42] & 72.34 & - \\
CRF [14] & 66.54 & - \\
AUEB [5] & 70.44 & - \\
MIN [38] & 73.44 & - \\
DE-CNN [12] & 74.37 & -
\end{tabular}

${ }^{1}$ http://alt.qcri.org/semeval2016/task5/ 


\begin{tabular}{llc}
\hline Model & Opinion target extraction & \\
\hline THA\&STN [37] & 73.61 & - \\
BiDTreeCRF [13] & 74.49 & - \\
Our CNN-IOB2 model & 99.96 & 99.93 \\
\hline
\end{tabular}

Table 2 shows that our proposed model outperforms the previous state-of-the-art methods for opinion target extraction subtask on $\mathrm{F} 1$ score metric and achieves outstanding results on Accuracy score metric.

F1_score: Our proposed model is $27.62 \%$ higher than NLANGP(U), 33.42\% higher than CRF model, $29.52 \%$ higher than AUEB model, 26.52\% higher than MIN model, 25.59\% higher than DE-CNN model, $26.35 \%$ higher than THA\&STN model and $25.47 \%$ higher than BiDTreeCRF model in the same Restaurant domain of the SemEval 2016 dataset.

Accuracy_score: Our model achieves pretty high result $99.93 \%$.
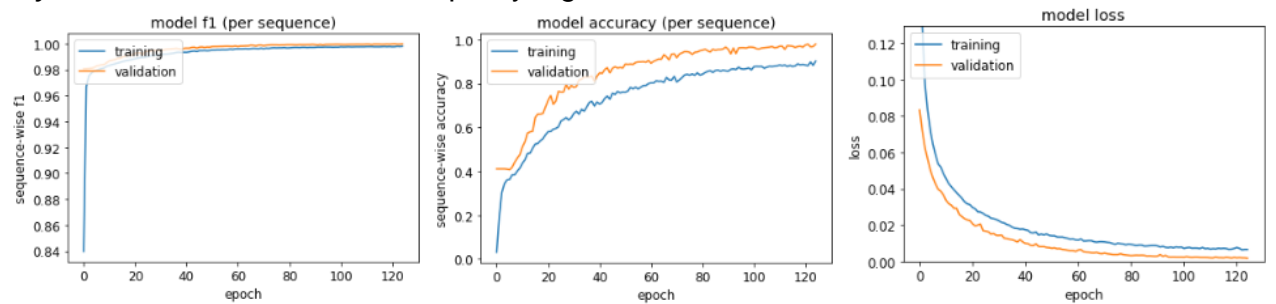

Figure 3: F1, Accuracy score and training loss vs. epoch curve of our opinion target extraction model

The graph from Figure 3 depicts our opinion target extraction model's validation F1, accuracy measure and loss. Observing the third graph_model loss in Fig. 3, we notice that after a sharp decline in the first few epochs and a gradual decrease in the next 40 epochs, the loss has a steady downtrend since the 60th epoch and reaches saturation state after the 100th epoch. In particular, the stability is evident from the 100th epoch to the 125th epoch.

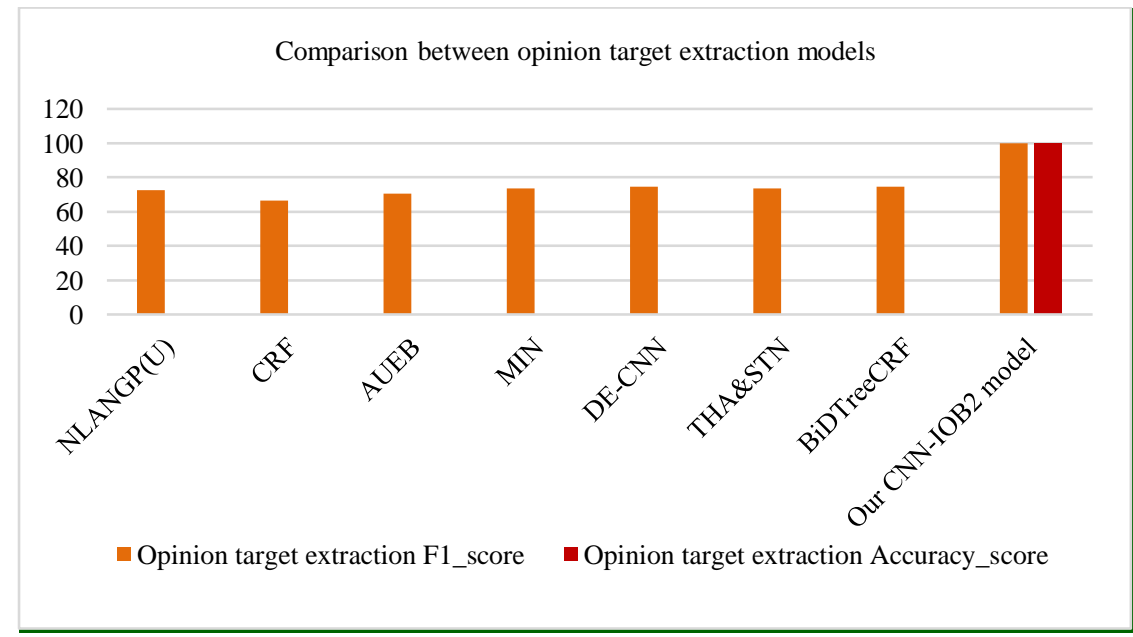

Figure 4: F1 \& Accuracy score of opinion target extraction models

As can be seen clearly from the graph in Figure 4, our proposed model achieves outstanding performance in both $F 1$ and accuracy scores over baseline models.

Table 3: Experimental results of our MABSA model in Precision, Recall, F1 and Accuracy_score on Restaurant domain of the SemEval 2016 dataset

\begin{tabular}{llll}
\hline Our MABSA model & Category classification & $\begin{array}{l}\text { Aspect } \\
\text { classification }\end{array}$ & $\begin{array}{l}\text { Sentiment Polarity } \\
\text { classification }\end{array}$ \\
\hline Precision_score & 97.97 & 92.52 & 97.19 \\
Recall_score & 97.67 & 92.24 & 96.58 \\
F1_score & 97.82 & 92.38 & 96.88 \\
Accuracy_score & 97.83 & 92.39 & 96.58 \\
\hline
\end{tabular}

Similar to our CNN-IOB2 model, our proposed MABSA model for remaining subtasks from Table 3. also shows good results between over $96 \%$ to approximately $98 \%$ for the two category and sentiment classification subtasks and over $92 \%$ for the aspect classification subtask on Precision, Recall, F1 and Accuracy score metrics. 

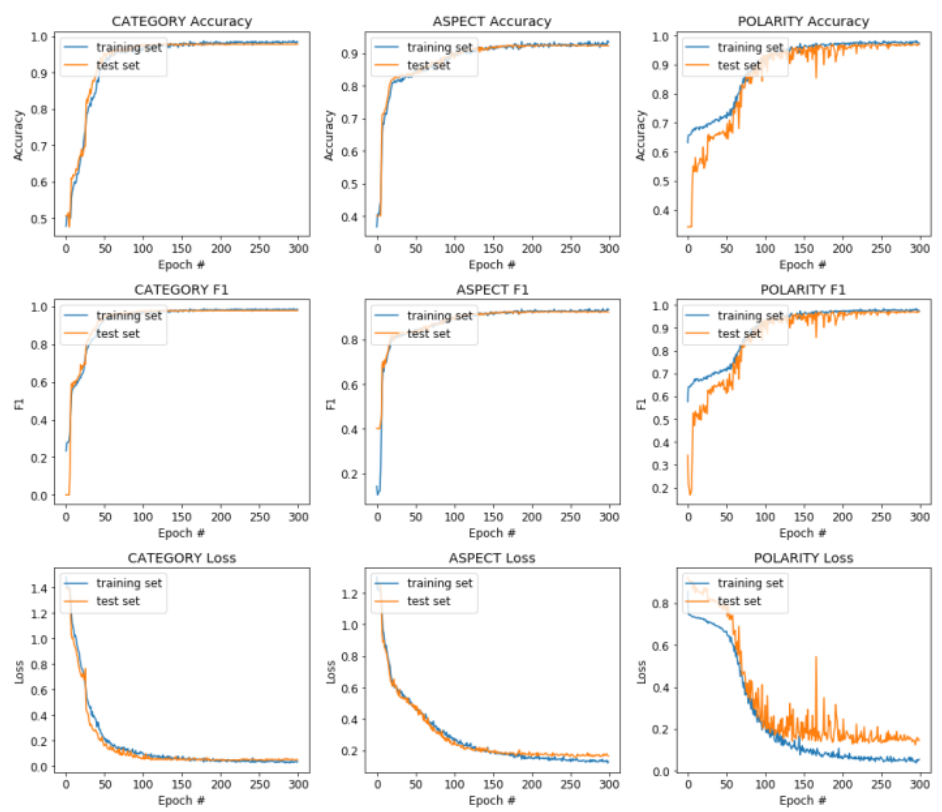

Figure 5: Precision, Recall, F1, Accuracy score and Loss of our MABSA model

In the graph cluster below from Figure 5, besides illustrating the performance on validation F1 and accuracy measures, we also can see the loss of the proposed model in the last three graphs. With category subtask, after a sharp decline in the first 50 epochs and a slight decrease from the 50th epoch to the 150th epoch, the loss tends to stabilize to the last epoch. The same goes for aspect loss graph, there is also a spike in the first 20 epochs, a smaller drop in the next 80 epochs, a slight drop from the 100th epoch to the 200th epoch and towards stabilized since the 200th epoch. The final polarity loss graph has a slight difference compared to the previous two graphs. After a drop in the first 50 epochs, there is a spike in the next 50 epochs and then stable from after the 100th epoch to the last 300th epoch.

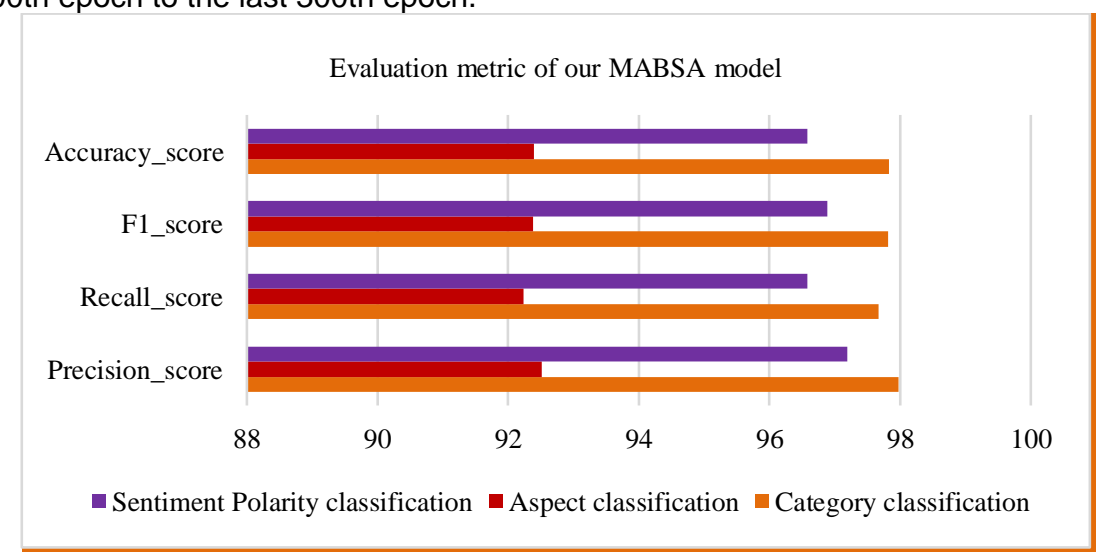

Figure 6: Precision, Recall, F1 \& Accuracy score of our MABSA model

Visually seen from the graph in Figure 6, our MABSA model shows the good performance across all three classification subtasks: category, aspect and sentiment polarity.

\section{CONCLUSION}

We have proposed a multitask deep learning approach to aspect based sentiment analysis. Our two models have finished main subtasks in ABSA: opinion target extraction, category classification, aspect classification and sentiment polarity classification used a three-layer CNN architecture that comprises the word embedding layer, GloVe and POS tagger to make the feature vectors for words in input opinion sentence. With the first model, we apply the IOB2 scheme on top of the CNN layers aims to label each token with one of three tags B, I or O for opinion target extraction subtask. Implementing the BiLSTM with one forward LSTM and one backward LSTM to take full advantage of information from both side of each word_the previous and the next tokens 
integrates with the three-layer CNN architecture mentioned above is used in the our second model. We apply a fully connected layer in the last state of the model and feed the output to the softmax layer to compute propability distribution over the possible categories for three remaining subtasks: category, aspect and sentiment classification. Our proposed models achieved significant improvement in performance over the previous state-of-the-art approaches. As future work, we plan to employ this our multitask learning model in multidomain dataset, the solution for domain adaptation.

\section{REFERENCES}

[1] Aurangzeb Khan, Baharum Baharudin. Sentiment classification using sentence-level semantic orientation of opinion terms from blogs. In 2011 National Postgraduate Conference, (Jan 23, 2012).

[2] Bing Liu. Sentiment Analysis and Opinion Mining. Morgan and Claypool Publishers, (May 2012).

[3] Binxuan Huang, Kathleen M. Carley. Parameterized Convolutional Neural Networks for Aspect LevelSentiment Classification. In Proceedings of the 2018 Conference on Empirical Methods in Natural Language Processing. Association for Computational Linguistics, Brussels, Belgium, (Oc 31 - Nov 4, 2018), 1091-1096.

[4] Dehong Ma, Sujian Li, Xiaodong Zhang, Houfeng Wang. Interactive Attention Networks for Aspect-Level Sentiment Classification. In arXiv: 1709.00893v1 [cs.Al], (Sep 4th 2017).

[5] Dionysios Xenos, Panagiotis Theodorakakos, John Pavlopoulos, Prodromos Malakasiotis, Ion Androutsopoulos. AUEB-ABSA at SemEval-2016 Task 5: Ensembles of Classifiers and Embeddings for Aspect Based Sentiment Analysis. In Proceedings of SemEval2016. Association for Computational Linguistics, San Diego, California, (Jun 16-17, 2016), 312-317.

[6] Duyu Tang, Bing Qin, Xiaocheng Feng, Ting Liu. Effective LSTMs for Target-Dependent Sentiment Classification. In arXiv: 1512.01100v2 [cs.CL], (Sep 29th 2016).

[7] Duyu Tang, Bing Qin, Ting Liu. Aspect Level Sentiment Classification with Deep Memory Network. In Proceedings of the 2016 Conference on Empirical Methods in Natural Language Processing. Association for Computational Linguistics, Austin, Texas, (Nov 1-5, 2016), 214224.

[8] Fangtao Li, Chao Han, Minlie Huang, Xiaoyan Zhu, Ying-Ju Xia, Shu Zhang, Hao Yu. Structure-aware review mining and summarization. In COLING'10 Proceedings of the $23^{\text {rd }}$ International Conference on Computational Linguistics: Posters, Beijing, (Aug 23-27, 2010), 653661.

[9] Gang Li, Fei Liu. Application of a clustering method on sentiment analysis. Journal of Information Science, Vol. 38, issue 2, (Feb 16th, 2012), 127-139.

[10] Guang Qiu, Bing Liu, Jiajun Bu and Chun Chen. 2011. Opinion word expansion and Target extraction through Double Propagation. Association for Computational Linguistics, Vol. 37, no. 1.

[11] Himabindu Lakkaraju, Richard Socher, and Chris Manning. 2014. Aspect specific sentiment analysis using hierarchical deep learning. In NIPS Workshop on Deep Learning and Representation Learning.

[12] Hu Xu, Bing Liu, Lei Shu, Philip S. Yu. Double Embeddings and CNN-based Sequence Labeling for Aspect Extraction. In Proceedings of the 56th Annual Meeting of the Association for Computational Linguistics (Short Papers). Association for Computational Linguistics, Melbourne, Australia, (Jul 15 - 20, 2018), 592-598.

[13] Huaishao Luo, Tianrui Li, Bing Liu, Bin Wang, Herwig Unger. Improving Aspect Term Extraction with BidirectionalDependency Tree Representation. In arXiv: 1805.07889v2 [cs.CL], (May 5th 2019).

[14] Hussam Hamdan. SentiSys at SemEval-2016 Task 5: Opinion Target Extraction and Sentiment Polarity Detection. In Proceedings of SemEval-2016. Association for Computational Linguistics, San Diego, California, (Jun 16-17, 2016), 350-355.

[15] Jaap Kamps, Maarten Marx, Robert J. Mokken, Maarten de Rijke. Using WordNet to measure semantic orientation of adjectives. In Proceedings of LREC-04, $4^{\text {th }}$ International Conference on Language Resources and Evaluation, Vol. 4, (Jan 2004), $1115-1118$.

[16] Jeffrey Pennington, Richard Socher, Christopher D. Manning. GloVe: Global Vectors for Word Representation. In EMNLP'14 Proceedings of the 2014 Conference on Empirical Methods in Natural Language Processing, Doha, (Oct 25-29, 2014), $1532-1543$.

[17] Maite Taboada, Julian Brooke, Milan Tofiloski, Kimberly Voll, Manfred Stede. Lexicon-Based Methods for Sentiment Analysis. In Computational Linguistics, Vol. 37, issue 2, (Jun 2011), 267-307.

[18] Meishan Zhang, Yue Zhang, Duy Tin Vo. Neural networks for open domain targeted sentiment. In Proceedings of the 2015 Conference on Empirical Methods in Natural Language Processing, Lisbon, (Sep 17-21, 2015), 612-621.

[19] Minqing Hu and Bing Liu. Mining opinion features in customer reviews. In AAAl'04 Proceedings of the $19^{\text {th }}$ national conference on Artificial Intelligence, (Jul 25-29, 2004), 755-760.

[20] Minqing $\mathrm{Hu}$ and Bing Liu. Mining and summarizing customer reviews. In KDD'04 Proceedings of the $10^{\text {th }}$ ACM SIGKDD international conference on Knowledge discovery and data mining, Seattle, WA, USA, (Aug 22-25, 2004), 168-177.

[21] Niklas Jakob and Iryna Gurevych. Extracting opinion targets in a single- and cross-domain setting with conditional random fields. In EMNLP'10 Proceedings of the 2010 Conference on Empirical Methods in Natural Language Processing, Cambridge, Massachusetts, (Oct 9-11, 2010), 1035-1045.

[22] Peng Chen, Zhongqian Sun, Lidong Bing, Wei Yang. Recurrent Attention Network on Memory for Aspect Sentiment Analysis. In Proceedings of the 2016 Conference on Empirical Methods in Natural Language Processing. Association for Computational Linguistics, Copenhagen, Denmark, (Sep 2017), 452-461.

[23] Peter D. Turney, Michael L. Littman. Measuring praise and criticism: Inference of semantic orientation from association. In ACM Transactions on Information Systems, Vol. 21, issue 4, (Oct 2003), 315-346.

[24] Po-Wei Liang and Bi-Ru Dai. Opinion Mining on Social Media Data. In IEEE 14th International Conference on Mobile Data Management, (Jul 30 $\left.{ }^{\text {th }}, 2013\right)$.

[25] Qiao Liu, Haibin Zhang, Yifu Zeng, Ziqi Huang, Zufeng Wu. Content Attention Model for Aspect Based Sentiment Analysis. In WWW '18: Proceedings of the 2018 World Wide Web Conference, (Apr 2018), 1023-1032.

[26] Ruidan He, Wee Sun Lee, Hwee Tou Ng, Daniel Dahlmeier. Effective Attention Modeling for Aspect-Level Sentiment Classification. In Proceedings of the 27th International Conference on Computational Linguistics, Santa Fe, New Mexico, USA, (Aug 20-26, 2018), 11211131. 
[27] Soujanya Poria, Erik Cambria, Alexander Gelbukh. Aspect extraction for opinion mining with a deep convolutional neural network. In Knowledge-Based Systems, Vol. 108, (Jun 2016), 42-49.

[28] Svetlana Kiritchenko, Xiaodan Zhu, Colin Cherry, Saif Mohammad. 2014. Detecting aspects and sentiment in customer reviews. In Proceedings of the 8th International Workshop on Semantic Evaluation (SemEval 2014), 437-442.

[29] Thien Hai Nguyen, Kiyoaki Shirai. Phrasernn. 2015. Phrase recursive neural network for aspect-based sentiment analysis. In Proceedings of the 2015 Conference on Empirical Methods in Natural Language Processing, 2509-2514.

[30] Tomas Mikolov, Kai Chen, Greg Corrado, Jeffrey Dean. Efficient Estimation of Word Representations in Vector Space. In arXiv:1301.3781v3 [cs.CL], (Sep $\left.7^{\text {th }}, 2013\right)$.

[31] Trang Uyen Tran, Ha Thanh Thi Hoang, Hiep Xuan Huynh. Aspect Extraction with Bidirectional GRU and CRF. In the 2019 IEEE-RIVF International Conference on Computing and Communication Technologies (RIVF), Danang, Vietnam, (Mar 20-22, 2019), 60-64.

[32] Trang Uyen Tran, Ha Thanh Thi Hoang, Hiep Xuan Huynh, "Bidirectional Independently Long Short-Term Memory and Conditional Random Field integrated model for Aspect Extraction in Sentiment Analysis", International Conference on Frontiers of Intelligent Computing: Theory and Applications (FICTA 2018), Advances in Intelligent Systems and Computing book series (AISC), Springer, Vol. 1014, (Oct 2, 2019), 131-140.

[33] Wei Xue, Tao Li. Aspect Based Sentiment Analysis with Gated Convolutional Networks. In Proceedings of the 56th Annual Meeting of the Association for Computational Linguistics. Association for Computational Linguistics, Melbourne, Australia, (Jul 15 - 20, 2018 ), 25142523.

[34] Wei Jin and Hung Hay Ho. A novel lexicalized HMM-based learning framework for web opinion mining. In ICML'09 Proceedings of the $26^{\text {th }}$ Annual International Conference on Machine Learning, Montreal, Quebec, (Jun 14-18, 2009), 465-472.

[35] Wenya Wang, Sinno Jialin Pan, Daniel Dahlmeier, Xiaokui Xiao. Recursive Neural Conditional Random Fields for Aspect-based Sentiment Analysis. In Proceedings of the 2016 Conference on Empirical Methods in Natural Language Processing. Association for Computational Linguistics, Austin, Texas, (Nov 1-5, 2016), 616-626.

[36] Xiaowen Ding, Bing Liu, Philip S. Yu. A holistic lexicon-based approach to opinion mining. In Proceedings of the 2008 International Conference on Web Search and Data Mining, (Feb 2008), 231-240.

[37] Xin Li, Lidong Bin, Piji Li, Wai Lam, Zhimou Yang. Aspect Term Extraction with History Attention and Selective Transformation. In Proceedings of the Twenty-Seventh International Joint Conference on Artificial Intelligence (IJCAI-18), (Jul 2018).

[38] Xin Li, Wai Lam. Deep Multi-Task Learning for Aspect Term Extraction with Memory Interaction. In Proceedings of the 2017 Conference on Empirical Methods in Natural Language Processing. Association for Computational Linguistics, Copenhagen, Denmark, (Sep 7-11, 2017), 2886-2892.

[39] Yequan Wang, Minlie Huang, Li Zhao, Xiaoyan Zhu. Attention-based LSTM for Aspect-level Sentiment Classification. In Proceedings of the 2016 Conference on Empirical Methods in Natural Language Processing. Association for Computational Linguistics, Austin, Texas, (Nov 1-5, 2016), 606-615.

[40] Yoon Kim. Convolutional Neural Networks for Sentence Classification. In Proceedings of the 2014 Conference on Empirical Methods in Natural Language Processing (EMNLP). Association for Computational Linguistics, Doha, Qatar, (Oct 25-29, 2014), 1746-1751.

[41] Yoshua Bengio, Patrice Simard, Paolo Frasconi. Learning long-term dependencies with gradient descent is difficult. In IEEE Transactions on Neural Networks, Vol. 5, issue 2. New York, USA, (Mar 1994), 157-166.

[42] Zhiqiang Toh, Jian Su. NLANGP at SemEval-2016 Task 5: Improving Aspect Based Sentiment Analysis using Neural Network Features. In Proceedings of SemEval-2016. Association for Computational Linguistics, San Diego, California, (Jun 16-17, 2016), pp. 282-288. 